\title{
Intersubband plasmon observation in electrochemically gated carbon nanotube films
}

Daria Satco $^{1 *}$, Daria S. Kopylova ${ }^{1}$, Fedor S. Fedorov ${ }^{1}$, Tanja Kallio ${ }^{2}$, Riichiro Saito ${ }^{3}$, Albert G. Nasibulin ${ }^{1,2 * *}$

${ }^{1}$ Skolkovo Institute of Science and Technology, Nobel str. 3, Moscow 143026, Russia

${ }^{2}$ Aalto University School of Chemical Engineering, Kemistintie 1, 02015, Espoo, 00076, Finland

${ }^{3}$ Department of Physics, Tohoku University, Sendai 980-8578, Japan

E-mail:*daria.satco@skoltech.ru;**a.nasibulin@skoltech.ru

\section{Supplementary Note 1. Optical absorption data}

Here we include additional figures (Figures S1 - S4) with optical spectra for each of the sample described in Table 1 of the main text. With increasing the applied potential, we observe suppression of $\mathrm{S}_{11}, \mathrm{~S}_{22}, \mathrm{M}_{11}$ peaks and appearance of intersubband plasmon. In the low-energy region of the spectra we can see the increase of optical absorption with doping, which is the contribution of Drude absorption and plasmons in the infrared and terahertz range. ${ }^{1,2}$

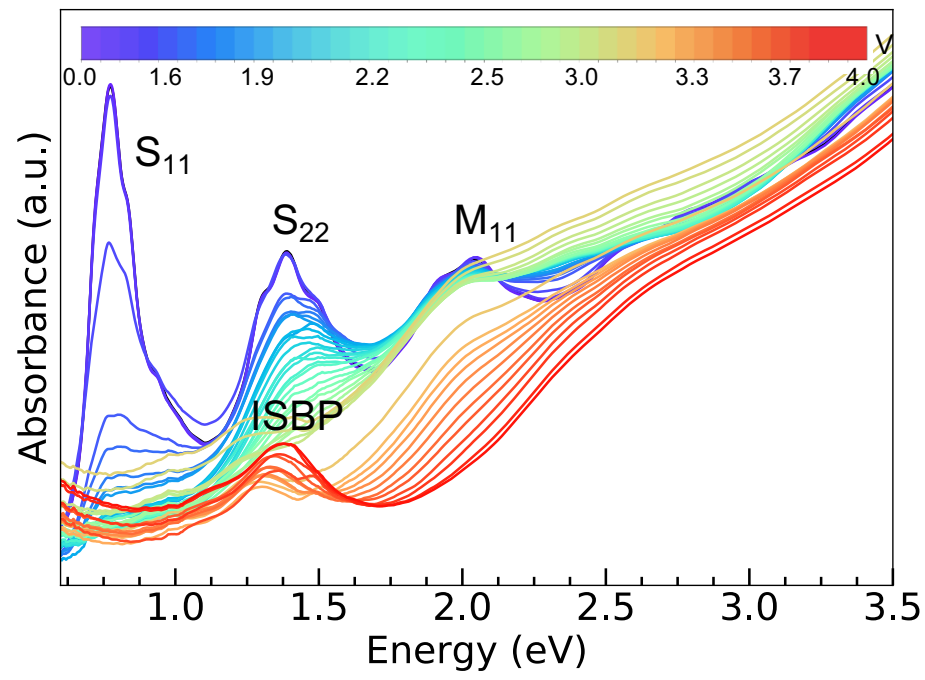

Figure S1. Evolution of the optical absorption spectra of electrochemically doped SWCNT film with $d_{t}=1.4 \mathrm{~nm}$ (sample $1.4 \mathrm{~nm}$ (bmim) S1 in Table 1). External potential is applied in the range from 0 to $4.0 \mathrm{~V}$. Ionic liquid absorption spectra is subtracted. 


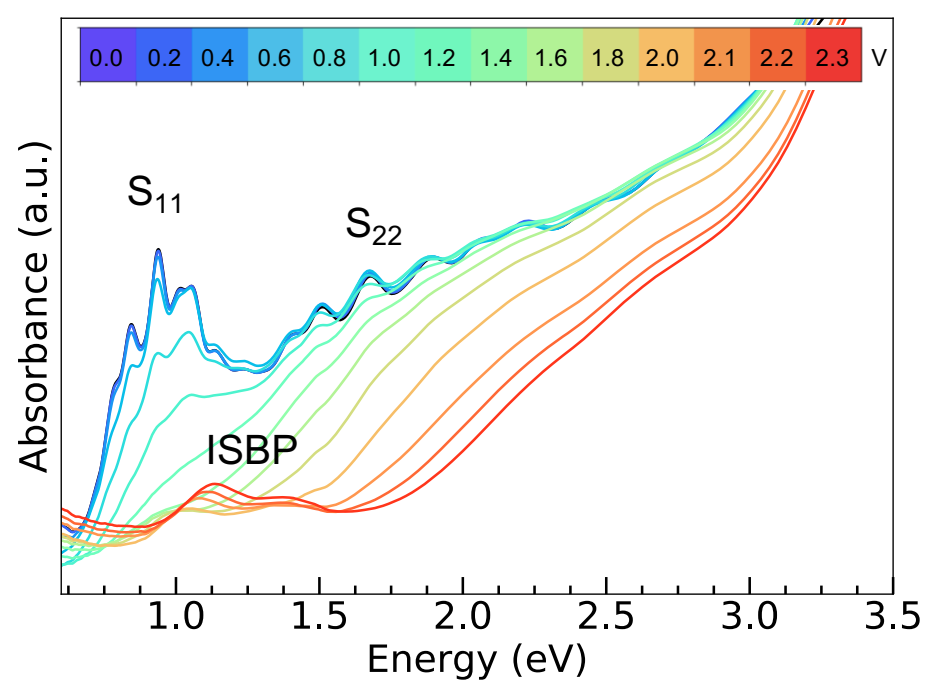

Figure S2. Evolution of the optical absorption spectra of electrochemically doped SWCNT film with $d_{t}=1.0 \mathrm{~nm}$ (sample $1.0 \mathrm{~nm}$ (deme) in Table 1). External potential is applied in the range from 0 to $2.3 \mathrm{~V}$. Ionic liquid absorption spectra is subtracted.

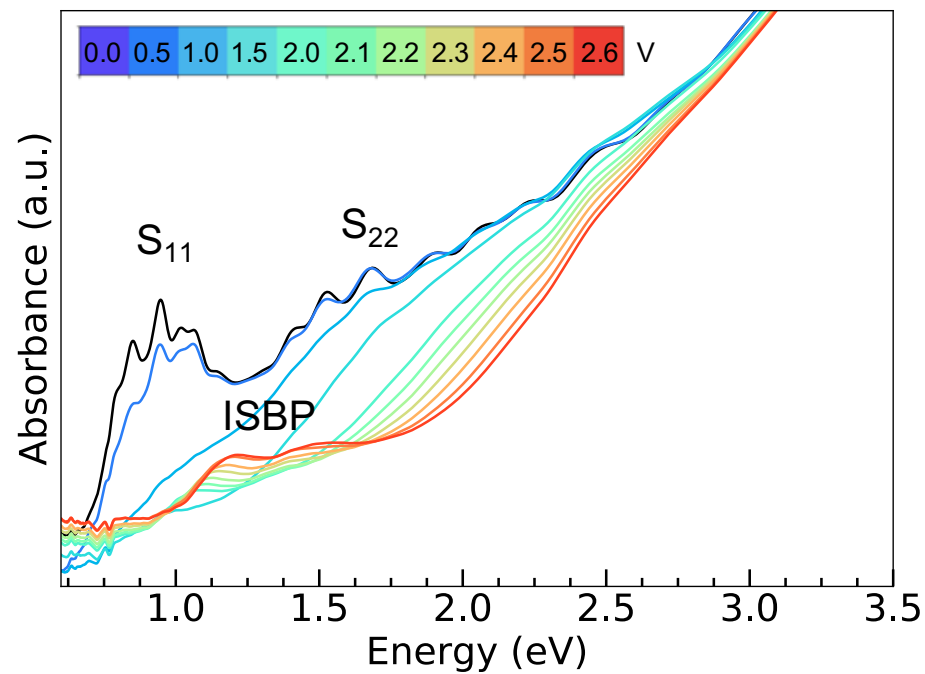

Figure S3. Evolution of the optical absorption spectra of electrochemically doped SWCNT film with $d_{t}=1.0 \mathrm{~nm}$ (sample $1.0 \mathrm{~nm}$ (bmim) in Table 1). External potential is applied in the range from 0 to $2.6 \mathrm{~V}$. Ionic liquid absorption spectra is subtracted.

Figures S2 and S3 show the optical absorption spectra of SWCNTs with $d_{t}=1.0 \mathrm{~nm}$. The intersubband plasmon splits into the two subpeak, which come from different chiral SWCNTs present in the film. Since the peak magnitude is proportional to the SWCNTs fraction within the sample, we consider the dominant plasmon peak position to build the dispersion function. 


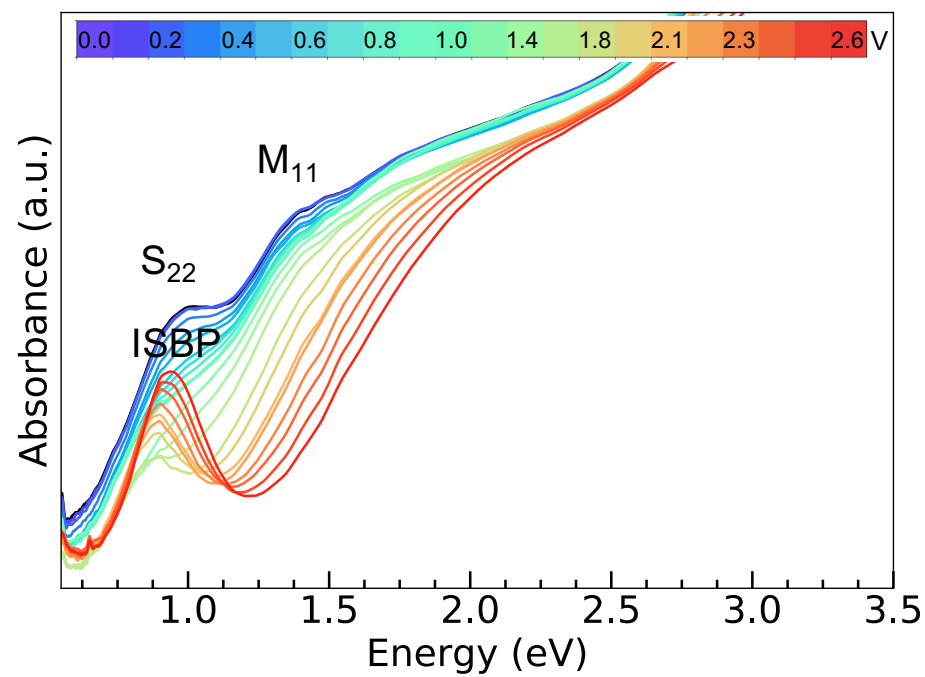

Figure S4. Evolution of the optical absorption spectra of electrochemically doped SWCNT film with $d_{t}=2.0 \mathrm{~nm}$ (sample $2.0 \mathrm{~nm}$ (deme) in Table 1). External potential is applied in the range from 0 to $2.6 \mathrm{~V}$. Ionic liquid absorption spectra is subtracted.

Figure S4 shows the optical spectra of the SWCNTs with $d_{t}=2.0 \mathrm{~nm}$, which were preliminary doped with gold. ${ }^{3}$ Extra-doping was used to suppress the $S_{11}$ transition, which results in the smaller potential needed to excite the ISBP.

\section{Supplementary Note 2. SWCNT doping}

In this note we explain how the applied potential is converted into the Fermi energy. Following the discussion from the Sec. Analysis of electrochemical gating, the conversion of the applied potential $U$ into the Fermi energy $E_{F}$ is done as: $E_{F}=U / \alpha$, where the parameter $\alpha$ is empirically found from the analysis of the optical spectra. Figure S5 (a) demonstrates the plasmon dispersion for the sample (S1) with $d_{t}=1.4 \mathrm{~nm}$ as a function of the applied potential and Fermi energy simultaneously (lower and upper $\mathrm{x}$-axis respectively). We choose $\alpha=2.5$, since it is consistent with the evolution of the peaks. By fitting the optical spectra at different applied potentials (see Figure S5 (b)-(g) and Sec. Spectrum analysis in the main text), we can capture the moment when each next optical transition is suppressed. When the ISBP appears for the first time $(U=2 \mathrm{~V})$, the $\mathrm{S}_{11}$ and $\mathrm{S}_{22}$ peaks are already fully suppressed, while the $\mathrm{M}_{11}$ is still present, which means that Fermi energy is lower than the $2^{\text {nd }}$ van Hove singularity of semiconducting SWCNT DOS. When the potential $U$ is around $2.4-2.5 \mathrm{~V}, \mathrm{M}_{11}$ peak almost disappears and the plasmon frequency changes its trend. This corresponds to the transition through the $1^{\text {st }}$ van Hove singularity of the metallic SWCNT DOS. Finally, at $U$ around 3.0 - 3.1 V we see the $\mathrm{S}_{33}$ is still excited in the spectra, which means that the Fermi level is higher than $-1.3 \mathrm{eV}$. Hence, fitting the spectra at different potentials and comparing it with DOS, we can choose a relevant parameter $\alpha$. This gives us an approximation of Fermi level as a function of the external potential. 

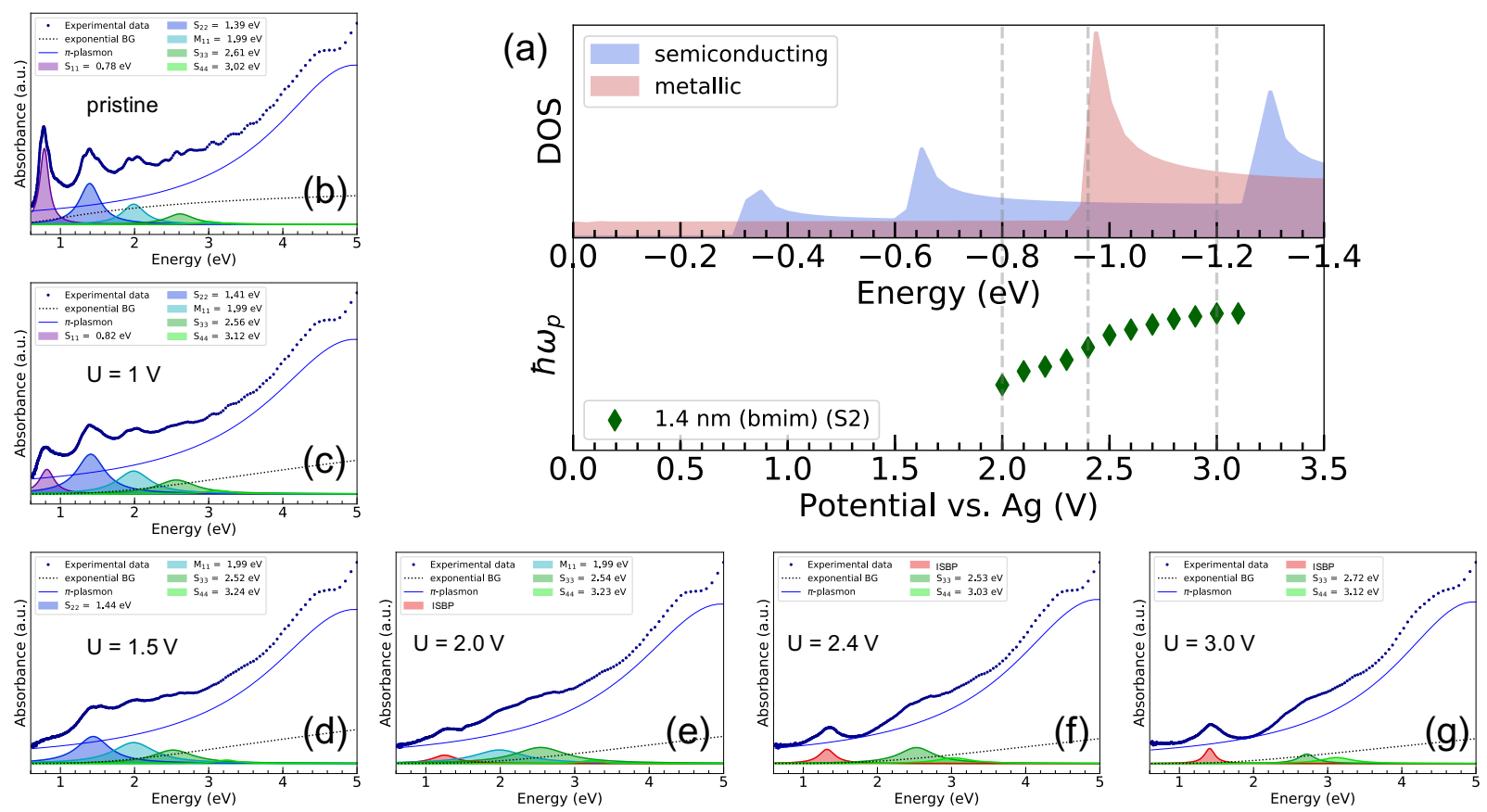

Figure S5. Estimation of the Fermi level shift with the applied potential. (a) Lower: plasmon dispersion for SWCNTs with $d_{t}=1.4 \mathrm{~nm}$ (sample (S2)). Upper: exemplary DOS for a single metallic $(10,10)$ and semiconducting $(14,6)$ SWCNT calculated within $3 \mathrm{NN}$ approach. ${ }^{4}(\mathrm{~b})-(\mathrm{g})$ fitting of the optical absorption spectra with Eq. 1 at several applied potentials.

Figure S6 demonstrates the ISBP dispersion and the corresponding DOS similar to the Figure S5 (a) to all other spectra. Following the same steps as discussed above we scale the applied potential with the best-fitted $\alpha$ (see Table S1). For the sample $1.0 \mathrm{~nm}$ (deme) we observe the peak before the $\mathrm{S}_{11}$ suppression, which is achievable in the case of the $p$-doping (see ref. 25 from the main text for details). For the sample with $d_{t}=2 \mathrm{~nm}$ we shift Fermi level lower than $3^{\text {rd }}$ van Hove singularity, so the $S_{33}$ is suppressed. The common feature of the dispersions is the presence of the kinks, which appear each time when the Fermi energy pass through the next van Hove singularity.

Table S1. Scaling parameters for the calculation of the Fermi energy.

\begin{tabular}{|l|c|}
\hline Sample & $\alpha$ \\
\hline $1.0 \mathrm{~nm}($ deme $)$ & 2.2 \\
\hline $1.0 \mathrm{~nm}(\mathrm{bmim})$ & 2.2 \\
\hline $1.4 \mathrm{~nm}(\mathrm{bmim}) \mathrm{S} 1$ & 2.5 \\
\hline $1.4 \mathrm{~nm}$ (bmim) $\mathrm{S} 2$ & 2.5 \\
\hline $2.0 \mathrm{~nm}$ (deme) & 2.8 \\
\hline
\end{tabular}


(a)

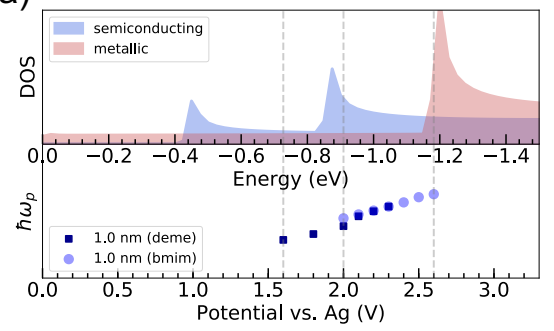

(b)

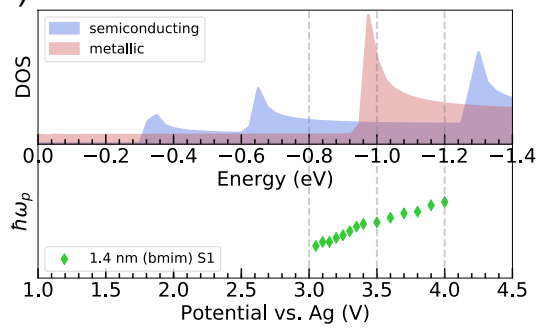

(c)

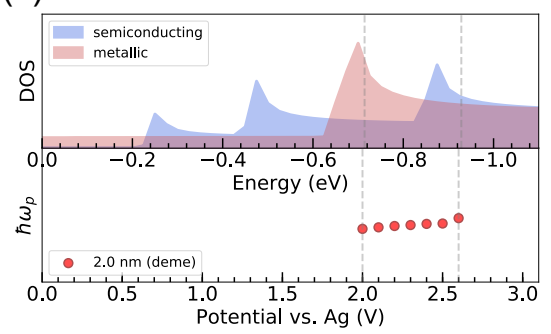

Figure S6. Estimation of the Fermi level shift with the applied potential. (a) Lower: plasmon dispersion for SWCNTs with $d_{t}=1 \mathrm{~nm}$. Upper: exemplary DOS for a single metallic $(8,8)$ and semiconducting $(8,7)$ SWCNT. (b) Lower: plasmon dispersion for SWCNTs with $d_{t}=1.4 \mathrm{~nm}$ (sample (S1)). Upper: exemplary DOS for a single metallic $(10,10)$ and semiconducting $(14,7)$ SWCNT. (c) Lower: plasmon dispersion for SWCNTs with $d_{t}=2 \mathrm{~nm}$. Upper: exemplary DOS for a single metallic $(23,5)$ and semiconducting $(22,6)$ SWCNT.

\section{Supplementary Note 3. Optical absorption spectra preprocessing}

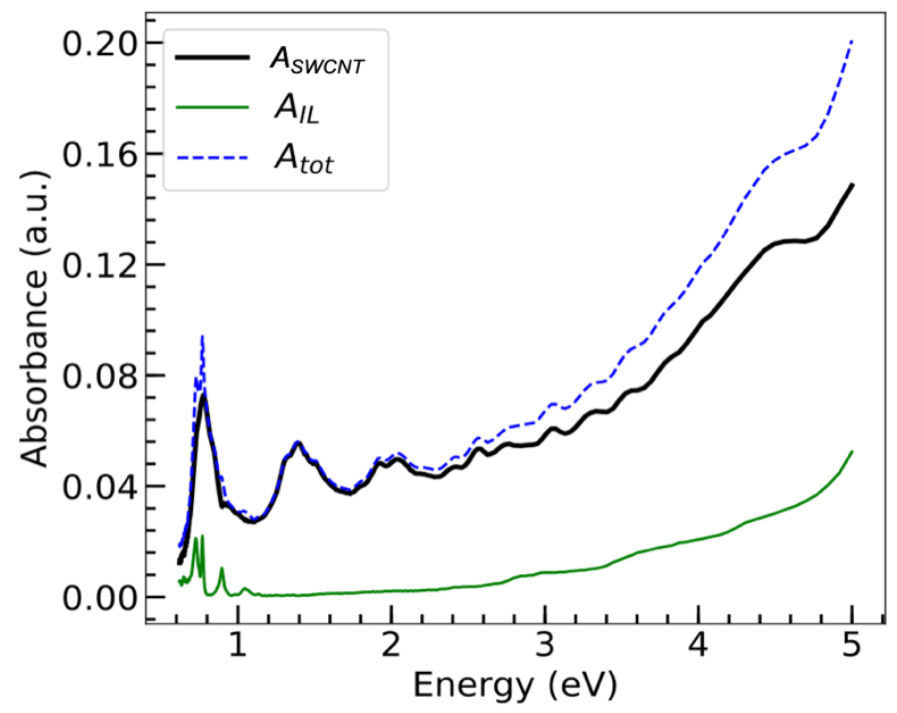

Figure S7. Experimental data preprocessing for SWCNTs with $d_{t}=1.4 \mathrm{~nm}$. Total optical absorption measured at $U=0 \mathrm{~V}$ (blue dashed line) is the sum of SWCNT and IL absorptions. Then the IL spectra (green solid line) is subtracted to obtain spectra from SWCNT (black line). 


\section{References}

(1) Chiu K-C.; Falk A. L.; Ho O-H.; Farmer D. B.; Tulevski G.; Lee Y-H.; Avouris P.; Han S-J. Strong and Broadly Tunable Plasmon Resonances in Thick Films of Aligned Carbon Nanotubes, Nano Lett. 2017, 17 (9), 5641-5645

(2) Zhukova E. S; Grebenko A. K.; Bubis A. V.; Prokhorov A. S.; Belyanchikov M. A.; Tsapenko A. P.; Gilshteyn E. P.; Kopylova D. S.; Gladush Yu. G.; Anisimov A. S.; Anzin V. B.; Nasibulin A. G.; Gorshunov B. P. Terahertz-infrared electrodynamics of single-wall carbon nanotube films. Nanotechnology 2017, 28 (44), 445204

(3) Tsapenko A. P.; Goldt A. E.; Shulga E.; Popov Z. I.; Maslakov K. I.; Anisimov A. S.; Sorokin P. B.; Nasibulin A. G. Highly conductive and transparent films of HAuCl4-doped single-walled carbon nanotubes for flexible applications. Carbon 2018, 130, 448-457

(4) Chegel R. Third-Nearest-Neighbors Tight-Binding Description of Optical Response of Carbon Nanotubes: Effects of Chirality and Diameter. Journal of Electronic Materials 2015, 44 (10), 3500-3511 